\title{
Bilayer order in a polycarbazole-conjugated polymer
}

Xinhui Lu ${ }^{1}$ Htay Hlaing ${ }^{1,2}$, David S. Germack${ }^{1}$, Jeff Peet ${ }^{3}$, Won Ho Jo ${ }^{4}$, Denis Andrienko ${ }^{5}$, Kurt Kremer ${ }^{5}$ \& Benjamin M. Ocko'

One of the best performing semiconducting polymers used in bulk heterojunction devices is PCDTBT, a polycarbazole derivative with solar-conversion efficiencies as high as $7.2 \%$. Here we report the formation of bilayer ordering in PCDTBT, and postulate that this structural motif is a direct consequence of the polymer's molecular design. This bilayer motif is composed of a pair of backbones arranged side-to-side where the alkyl tails are on the outer side. This is in stark contrast to the monolayer ordering found in other conjugated polymers. The crystalline bilayer phase forms at elevated temperatures and persists after cooling to room temperature. The existence of bilayer ordering, along with its high-packing fraction of conjugated moieties, may guide the synthesis of new materials with improved optoelectronic properties.

\footnotetext{
${ }^{1}$ Condensed Matter Physics and Materials Science Department, Brookhaven National Laboratory, Upton, New York 11973, USA. ${ }^{2}$ Department of Physics and Astronomy, State University of New York, Stony Brook, New York 11794, USA. ${ }^{3}$ Konarka Technologies, Lowell, Massachusetts 01852, USA. ${ }^{4}$ Department of Materials Science and Engineering, Seoul National University, Seoul 151-742, Korea. ${ }^{5}$ Max Planck Institute for Polymer Research, Ackermannweg 10, 55128 Mainz, Germany. Correspondence and requests for materials should be addressed to B.M.O. (email: ocko@bnl.gov).
} 
C onjugated polymers have been proposed for use in a wide variety of next generation technologies including displays, solid-state lighting, transistors and organic photovoltaic devices $^{1-3}$, but relatively little is known about how these polymers pack with the exception of a few materials ${ }^{4}$. The active layer in organic photovoltaic bulk heterojunction devices is composed of a blend of $\pi$-conjugated, electron-donor polymers and electronacceptor molecules, such as the fullerene derivative [6,6]-phenylC61-butyric acid methyl ester (PCBM). Whereas the optoelectronic properties of the electron-donor polymer are primarily determined by the choice of the conjugated backbone, the solubility is predominantly determined by the position, length and makeup of the alkyl side chains. Both the polymer backbone and the alkyl side chains influence device efficiency and molecular packing structure, especially in donor-acceptor copolymers, which contain an ordered sequence of different subunits ${ }^{5}$. Systematic studies of the effect of different side-chain groups, with the same backbone, have shown that the length and the type of alkyl chains affect the packing structure of the active layer, such as the $\pi-\pi$ distance and polymer orientation, and these properties have been correlated with the device performances, fill factor, open circuit voltage and short circuit current. Such studies have been conducted for alternating siliconbridged bithiophene and benzothiadiazole ${ }^{6}$, alternating thienothiophene and benzodithiophene $(\mathrm{PTB})^{7}$, alternating naphtho[2, 1-b:3,4-b']dithiophene and 4,7-di(thiophen-2-yl)benzothiadiazole $(\text { PNDT-DTBT })^{8}$, alternating bithiazole and benzodithiophene $(\mathrm{BTz}-\mathrm{BDT})^{9}$, and poly(3-alkylthiophene) (P3AT) ${ }^{10}$ among others. Decreasing the length (volume) of the alkyl chains has the potential to improve the polymer's charge mobility and optical density because these regions are resistive and do not contribute to optical absorption, but steric and other factors may lead to the opposite behaviour ${ }^{10}$.

The molecular structure of poly[ $N$ - $9^{\prime \prime}$-hepta-decanyl-2,7-carbazole-alt-5,5-( $4^{\prime}, 7^{\prime}$-di-2-thienyl-2', $1^{\prime}, 3^{\prime}$-benzothiadiazole $\left.)\right]$ (PC DTBT), is shown in Fig. 1a. First synthesized by Leclerc and coworkers $^{11}$, it is one of the most extensively studied donor-acceptor copolymers. The lone branched side chain along the $\sim 2 \mathrm{~nm}$ backbone subunit-repeat distance is unusual as most polymers with such long repeat lengths have multiple alkyl chains with more alkyl chain material per polymer repeat unit. PCDTBT has been reported to have relatively high solubility ${ }^{11}$, nearly perfect internal quantum efficiency $^{11}$, high-power conversion efficiency $(>7.2 \%)^{12,13}$ in samples annealed below $80^{\circ} \mathrm{C}$ and excellent thermal stability ${ }^{14}$, with operating lifetimes estimated to be $\sim 7$ years ${ }^{15}$. For thin films, no crystalline peaks were detected in the X-ray scattering profiles in studies (conventional source), for samples annealed as high as $400^{\circ} \mathrm{C}$ (ref. 14). On this basis, an amorphous structure was reported. However, bulk PCDTBT showed the existence of two broad features, attributed to a layer spacing of $20.9 \AA$ and a $\pi-\pi$ stacking distance of $4.4 \AA$ (ref. 16), much greater than the reported $\pi-\pi$ distances of other successful polymers, such as $3.8 \AA$ for Poly(3-hexylthiophene) $(\mathrm{P} 3 \mathrm{HT})^{17,18}$, and $3.55 \AA$ for Poly[2,1,3-benzothiadiazole4,7-diyl[4,4-bis(2-ethylhexyl)-4H-cyclopenta[2,1-b:3,4-b'] dithiophene-2,6-diyl]] (PCPDTBT) ${ }^{19}$. For many systems, a large $\pi-\pi$ distance will hinder the electronic coupling and lead to poor device performance ${ }^{7}$. Despite the large $\pi-\pi$ spacing and the reported amorphous-like structure, PCDTBT photovoltaic devices with thin active layers exhibit excellent performance. Additional structural features of PCDTBT, which may help provide a basis for the superior performance, have not been previously reported.

\section{Results}

X-ray scattering studies of PCDTBT films. Thin films, $\sim 100 \mathrm{~nm}$ thick, made from PCDTBT were investigated, using Grazing incidence wide-angle X-ray scattering (GIWAXS) (Fig. 1b) under vacuum. To provide an overview of the scattering from the

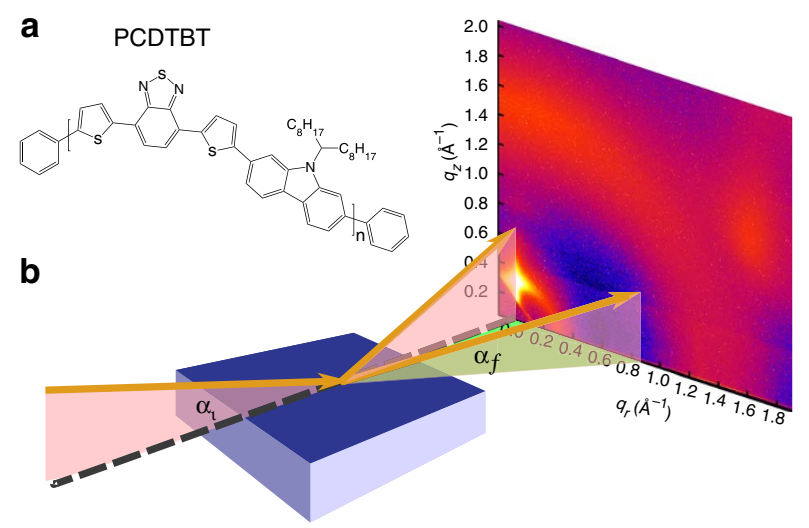

Figure 1 | The chemical structure of the PCDTBT polymer and a schematic of the X-ray scattering geometry. (a) The molecular structure of PCDTBT. (b) The experimental geometry of the grazing incidence wide-angle $\mathrm{X}$-ray scattering geometry.

PCDTBT thin films, we show 4 PCDTBT scattering patterns in Fig. 2 at (a) $25^{\circ} \mathrm{C}$, (b) $140{ }^{\circ} \mathrm{C}$, (c) $200^{\circ} \mathrm{C}$, and (d) after subsequent cooling to $25^{\circ} \mathrm{C}$. At $25^{\circ} \mathrm{C}$, before annealing (Fig. 2a), two weak and broad diffusive rings of scattering were observed along with a vertical streak that is associated with specular diffuse scattering. The PCDTBT scattering features, shown in Fig. 2a, were more defined than those reported previously under similar sample preparation conditions, using a much weaker, conventional X-ray source ${ }^{12}$. In both previous and present studies, the diffuse nature of the scattering indicates a rather disordered, amorphous-like state of the polymer film. For P3HT, a prototypical conjugated polymer, similar scattering rings, albeit somewhat better defined, have been associated with the distance between layers and to the $\pi-\pi$ stacking distance ${ }^{1}$. By analogy, we also associate the ring at about $0.35 \AA^{-1}$ in PCDTBT as originating from the layer spacing and the ring at $1.42 \AA^{-1}$ with the $\pi-\pi$ stacking distance. From Fig. $2 \mathrm{a}$, the layering ring appears slightly elliptical-like rather than circular as it is located at $\sim 0.36 \AA^{-1}$ along the $q_{z}$ axis, and at $\sim 0.33 \AA^{-1}$ along the $q_{r}$ axis. These peak positions respectively correspond to layer spacings of $\sim 17.5$ and $\sim 19.0 \AA$, indicating that the layer spacing is more compact when the layers stack along the $q_{z}$ (surface normal) direction, thus suggesting coexisting polymorphs. Coexisting polymorphs have also been observed in similar thin films materials such as PCPDTBT ${ }^{20}$. The observed PCDTBT layer spacings are smaller than the $20.9 \AA$ spacing reported for bulk PCDTBT ${ }^{16}$. The $\pi-\pi$ stacking ring $\left(q \sim 1.42 \AA^{-1}\right)$ corresponds to an average spacing of $4.4 \AA$, identical to that obtained for bulk samples ${ }^{16}$.

The scattering pattern at $140^{\circ} \mathrm{C}$ (Fig. 2b) exhibits similar features as to the $25^{\circ} \mathrm{C}$ pattern. However, the scattering ring associated with layering is both sharper and more intense than at $25^{\circ} \mathrm{C}$, suggesting enhanced order. The layering peak is somewhat aligned along the $q_{z}$ direction, thus suggesting a preferential 'edge-on' orientation. In addition, this scattering ring is now symmetric with a uniform radius of $0.302 \AA^{-1}$, corresponding to a $20.8 \AA$ layer spacing. This seems to suggest that the multiple polymorphs observed at lower temperatures have vanished. There is no apparent change in the peak positions and widths associated with the $\pi-\pi$ stacking along the $q_{z}$ direction between 25 and $140^{\circ} \mathrm{C}$; however, the intensity gradually increases with increasing temperature. At $140{ }^{\circ} \mathrm{C}$, above the $130{ }^{\circ} \mathrm{C}$ glass-transition temperature ${ }^{16}$, weak features start to emerge along the $q_{z}$ axis between the layering and the $\pi-\pi$ peaks. These diffraction features correspond to the onset of a crystalline structure that is fully established by $200^{\circ} \mathrm{C}$, as discussed below. 

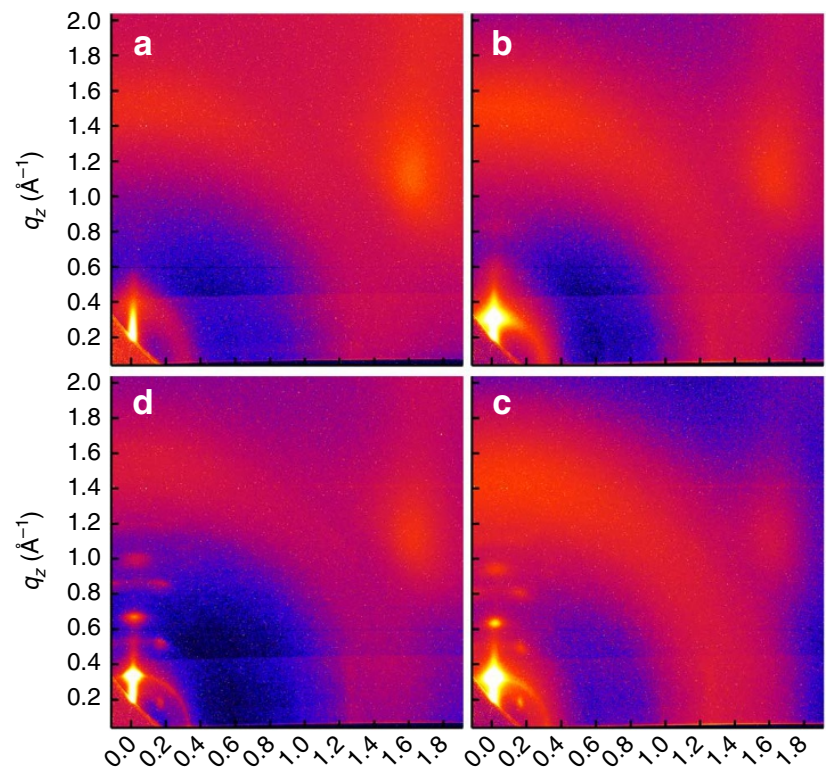

$q_{r}\left(\AA^{-1}\right)$

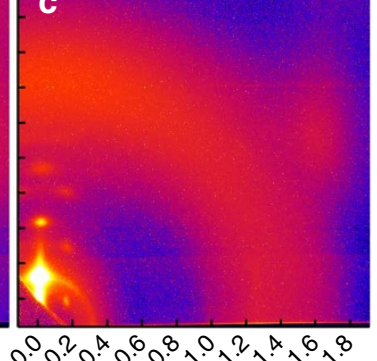

$q_{r}\left(\AA^{-1}\right)$

Figure 2 | GIWAXS scattering images of PCDTBT. The images were obtained at (a) $25^{\circ} \mathrm{C}$ before annealing; (b) $140{ }^{\circ} \mathrm{C}$; (c) $200^{\circ} \mathrm{C}$; (d) $25^{\circ} \mathrm{C}$ after annealing.

Well-defined Bragg peaks are observed at $200^{\circ} \mathrm{C}$ (Fig. 2c) thus indicating the formation of a crystalline phase. We note that previous studies, albeit with a much weaker source (non-synchrotron), on PCDTBT films at similar temperatures failed to reveal the formation of a crystalline or oriented phase ${ }^{12}$. The diffraction peaks remain even after the film was cooled to $25^{\circ} \mathrm{C}$ (Fig. 2d) and this provides strong evidence that the amorphous structure observed before annealing is a non-equilibrium structure. The layering peak at $\sim 0.320 \AA^{-1}$ along the $q_{z}$ axis, also indicating preferential 'edge-on' orientation, appears very similar to that observed at $140^{\circ} \mathrm{C}$, whereas the new spots at $\sim 0.634$ and $\sim 0.947 \AA^{-1}$, which emerge at around $160^{\circ} \mathrm{C}$ (shown in Fig. 3), were not observed at $140^{\circ} \mathrm{C}$. The two new peaks are second- and third-order reflections; thus, the overall layering motif with a $\sim 20 \AA$ layer spacing does not change between 140 and $200^{\circ} \mathrm{C}$. The appearance of the six off-axis peaks indicates the formation of long-range order structure parallel to the substrate and by $200^{\circ} \mathrm{C}$, they are located at $( \pm 0.150,0.186),( \pm 0.150,0.494)$ and $( \pm 0.150,0.811) \AA^{-1}$. This long-range order must originate from crystallinity along the backbone direction as (1) the scattering associated with the $\pi-\pi$ stacking is virtually unchanged between 140 and $200^{\circ} \mathrm{C}$ and $(2)$ the length scale $2 \pi / 0.150 \AA^{-1} \approx 41.9 \AA$ is too long to be along the $\pi-\pi$ stacking direction. The scattering pattern includes contributions from a crystalline phase with its well-defined Bragg peaks and a non-crystalline phase that gives rise to the more diffuse scattering features. These diffuse features do not affect the overall identification of the structure of the crystalline regions, the focus of the present investigation. Films prepared both by bladecoating and spin-casting, provided similar results and indicated no preferential backbone alignment with respect to the blade-coating direction.

Unit cell calculation and the bilayer motif. The diffraction pattern corresponding to the observed reflections is illustrated in Fig. 4a. We note that the pattern cannot be indexed by a rectangular or centred-rectangular unit cell, but rather by an oblique unit cell with $b_{1}=(0,0.333) \AA^{-1}$ and $b_{2}=(0.150,0.190) \AA^{-1}\left(25^{\circ} \mathrm{C}\right.$, after annealing). The real-space primitive unit cell vectors are $a_{1}=(-23.9,18.9)$ and $a_{2}=(41.9,0) \AA$ (Fig. $\left.4 \mathrm{~b}\right)$, where the $x$ axis is

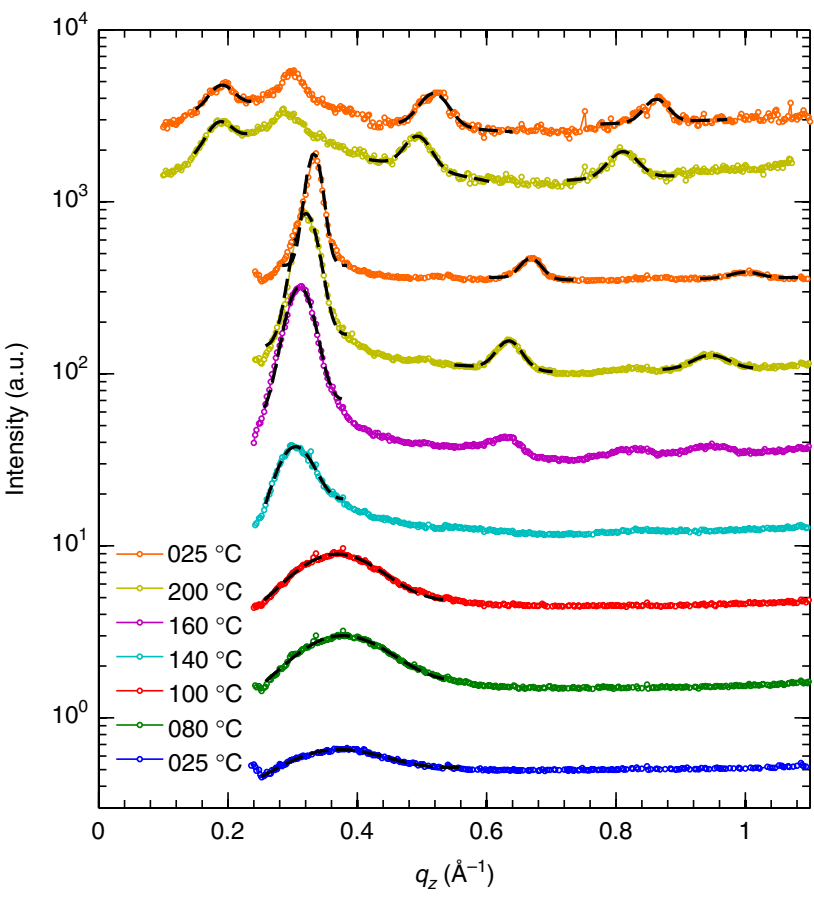

Figure 3 | PCDTBT scattering profiles along $q_{z}$ at various temperatures. The scattering profiles along $q_{z}$ obtained at $q_{r} \sim 0$ (lower seven curves) and $q_{r}=0.15 \AA^{-1}$ (top two curves). The dashed lines (black) are fits to a Gaussian profile. The blue $25^{\circ} \mathrm{C}$ curve is measured before thermal treatment. The orange $25^{\circ} \mathrm{C}$ curve is measured after thermal treatment.

in-plane and the $y$ axis is out-of-plane. The $41.9 \AA$ length corresponds to the length of two linear backbone segments. Further, there is a constant shift of $23.9 \AA$ between backbone chains separated by the $18.9 \AA$ A layer spacing. In Fig. 4c, we show the proposed molecular motif of the lamellar stacking structure of PCDTBT, which includes the pairing of neighbouring conjugated backbones to form bilayers (discussed below). In the 'molecular schematic', the alkyl chains are not explicitly drawn because there is no evidence based on the diffraction or Fourier transform infrared spectroscopy (FTIR) (Supplementary Fig. S1) of chain crystallinity, and because the chains are not confined to a single layer owing to the high density of gauche defects $^{21,22}$. Figure $4 \mathrm{c}$ shows that the undulations between neighbouring bilayers are out-of-phase with each other and this allows the alkyl chains to aggregate giving the appearance of a chain of alkyl beads. As only short-range order is observed along the $\pi-\pi$ stacking direction, there is no evidence for three-dimensional crystallization.

Packing and mass density considerations, as described below, clearly show that there must be four PCDTBT repeating units per unit cell, and this provides support for the pairing model shown in Fig. 4c. To determine the room temperature mass density, we calculated the volume of the unit cell to be $3,484 \AA^{3}$, using the measured two-dimensional (2D) unit cell lattice parameters (18.9 and $41.9 \AA$ ) along with the average $\pi-\pi$ stacking distance ( $4.4 \AA$ ). Placing two segments $(\mathrm{MW}=2 \times 701)$ into the unit cell gives rise to a density of $0.67 \mathrm{~g} \mathrm{~cm}^{-3}$. If there are 4 PCDTBT segments per unit cell, the calculated density doubles to $1.34 \mathrm{~g} \mathrm{~cm}^{-3}$. Although direct density measurements were inaccurate because of bubble formation, the density is expected to be higher than the mass density of P3HT $\left(1.10 \mathrm{~g} \mathrm{~cm}^{-3}\right)^{17}$. A centred arrangement formed by including an additional polymer backbone between the layers is inconsistent with the diffraction pattern, as this centred lattice would give rise to missing diffraction peaks that are observed. This indicates that the layers are composed of conjugated backbone pairs, rather than 
a

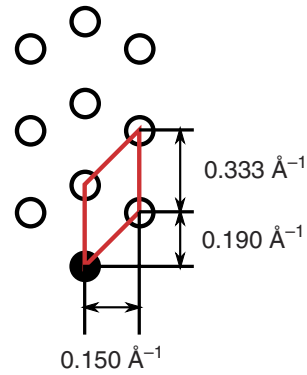

b

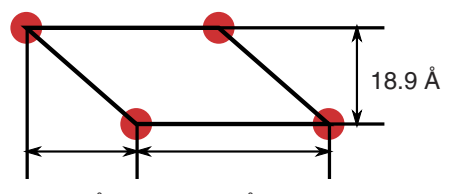

$23.9 \AA \quad 41.9 \AA$ d

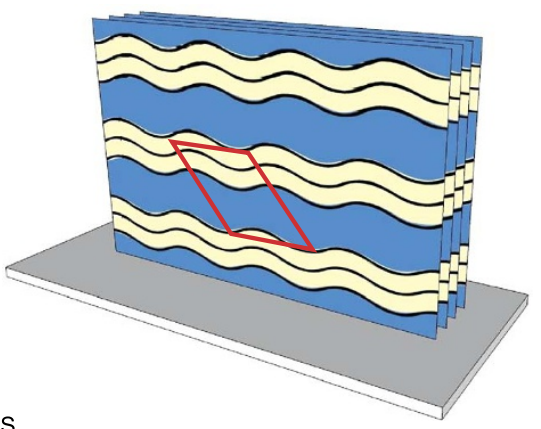

C

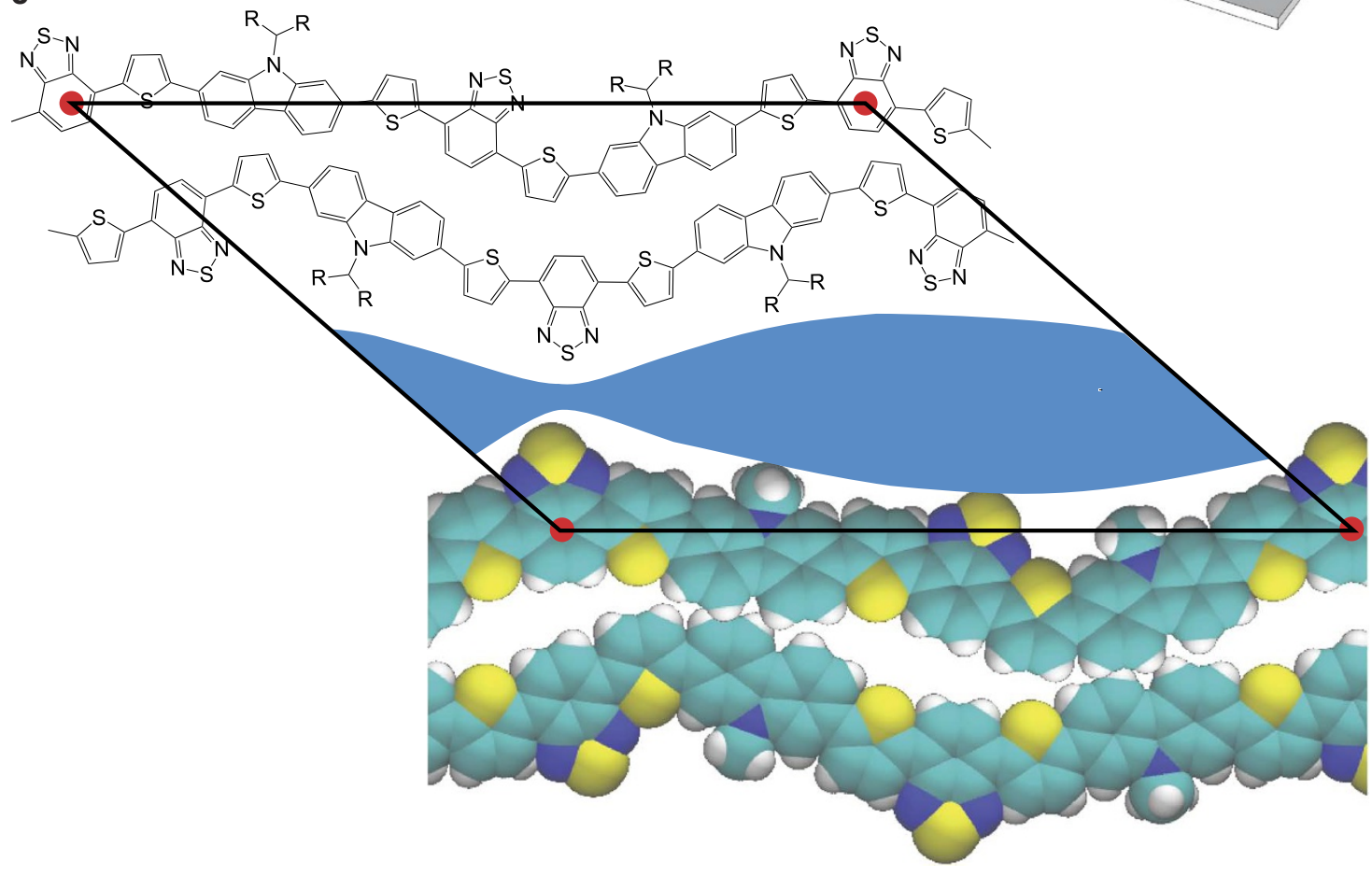

Figure 4 | PCDTBT thin-film diffraction pattern, unit cell and bilayer motif. (a) Diffraction pattern obtained from the Bragg peaks shown in Fig. 2d. The filled circle corresponds to the origin. (b) The corresponding oblique real-space lattice obtained from the scattering pattern. The $\pi$ - $\pi$ direction is normal to this plane. (c) Undulating backbone configuration for one of possible conformations of a backbone obtained by ab initio calculations (Fig. 5c). The second chain is merely a rotated/translated copy of the first one. Including the full interactions would provide further adjustment in the positions perpendicular to the backbone. The alkyl chains $\left(\mathrm{C}_{8} \mathrm{H}_{17}\right)$ are denoted by $\mathrm{R}$ for simplicity and occupy the blue-shaded region. This is the same oblique unit cell shown in (b).

(d) A three-dimensional illustration of the crystalline phase where the red trapezoid identifies the 2D unit cell.

a single backbone as with P3HT, thus supporting the bilayer arrangement shown in Fig. 4c,d.

In the following, we introduce a simple method of calculating the absolute volume contributions from the alkyl and the conjugated regions into the unit cell of conjugated polymers and, as described below, this approach supports the monolayer designation for P3HT and the bilayer designation for PCDTBT. For each polymer repeat segment, we assign atoms in the two octyl chains to the alkyl region and the remaining atoms to the conjugated region. The thickness of the alkyl region is calculated from the ratio of the alkyl volume (calculated from its bulk $n$-alkane values) to the cross-sectional area of the unit cell transverse to the layering direction, where we have assumed that the alkyl chains are space filling. For PCDTBT, this transverse cross-sectional area is $41.9 \times 4.4 \AA$ and each octyl chain has a volume of $240 \AA^{3}$, half the volume of a liquid $n$-hexadecane molecule. If we assume a monolayer motif (two PCDTBT segments per unit cell), an alkyl region layer thickness of $5.2 \AA$ is obtained. A conjugated region layer, with a thickness of $13.7 \AA$, is obtained from the difference between the $18.9 \AA$ layer spacing and the $5.2 \AA$ alkyl region layer thickness. This is more than 3 times the $4.1 \AA$ P3HT conjugated thickness obtained using a similar calculation taking into account a layer spacing of $16.8 \AA$ and a $\pi-\pi$ stacking distance of $3.8 \AA$ for P3HT (ref. 17). For the proposed PCDTBT bilayer model (4 PCDTBT segments per unit cell) shown in Fig. 4c, the calculated alkyl region layer thickness is $2 \times 5.2=10.4 \AA$, thus leaving a conjugated thickness of $8.5 \AA$ for a pair of backbone segments, a reasonable thickness compared with the unphysical, $13.8 \AA$ thickness calculated for the monolayer model above. This conjugated region thickness is about twice the calculated $4.1 \AA$ P3HT thickness (see above). Finally, for the proposed pairing model shown in Fig. 4c,d there is a sinusoidal-like variation in the position of the backbone along the layering direction and this feature explains the absence of higher order diffraction peaks along $q_{r}$.

First principles calculation of backbone conformations. The precise details of the pairing model can not be ascertained from the $\mathrm{X}$-ray data alone, because there are too few reflections to provide a crystallographic analysis. Additional structural insight on the 
A

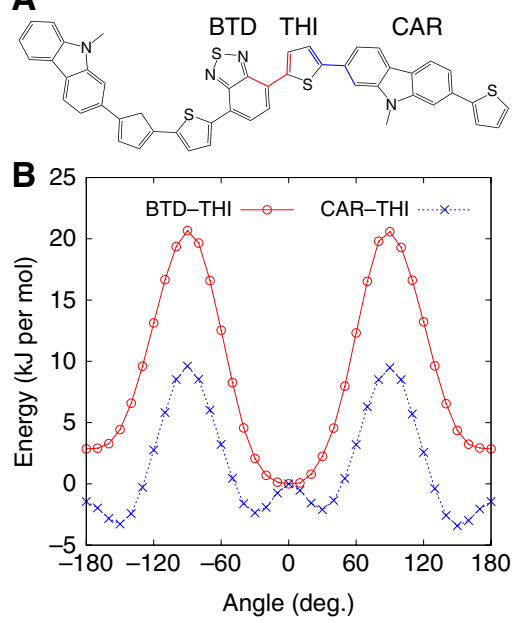

C
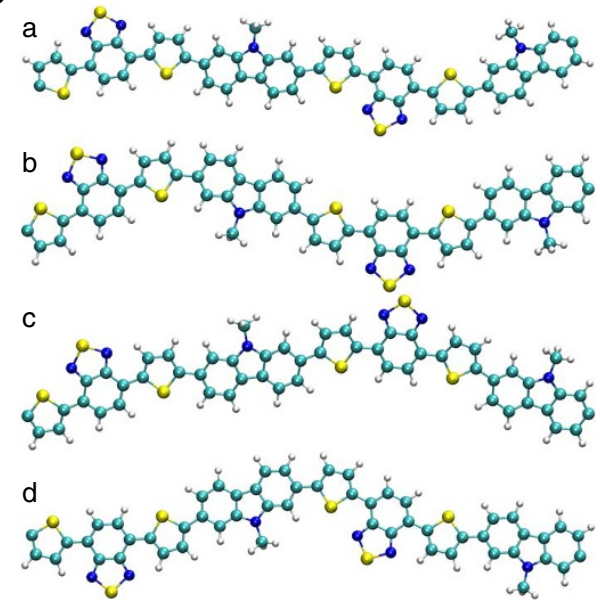

Figure 5 | Backbone conformations obtained from first principles calculations. (A) Scanned dihedrals: the shown BTD-THI (red) dihedral angle is $\phi=0$ and CAR-THI (blue) angle is $\phi=\pi$. (B) Potential energy surfaces of scanned dihedrals. (C) Planar molecular conformations optimized in periodic boundary conditions.

backbone configuration was obtained from the $a b$ initio calculations described below. All calculations were performed using the GAUSSIAN 09 package ${ }^{23}$. The dimer (two backbone repeat units) of PCDTBT with a methyl side chain (Fig. 5A) was first optimized using the B3LYP functional and the 6-311g(d) basis set. The potential energy surfaces of the two dihedrals (benzothiadiazole (BTD)-thiophene(THI) and carbazole(CAR)-THI) were then scanned using the same functional and basis set. The results, shown in Fig. 5B, indicate that the BTD and the THI units prefer to have dihedral angle of $\phi=0$, though the second minimum of $\phi=\pi$ is only $2.9 \mathrm{~kJ}$ per mol $\left(1.15 k_{\mathrm{B}} \mathrm{T}\right.$ at room temperature $)$ higher. The $\pi$-systems of the CAR and the THI repeat units are not parallel to each other, also the two minima at $\sim 30$ and $150^{\circ}$ have practically the same energy. One can conclude that there is practically no preference for the backbone to have trans- or cis-conformations between the repeat units, and self-assembly is mostly driven by the competition between packing of side chains and van der Waals interactions between backbones.

The backbone conformation within the proposed unit cell was also calculated. The dimer (Fig. 5A) has seven dihedral angles and hence $2^{7}$ distinct conformers. As a $180^{\circ}$ flip of the BTD group does not practically change the polymer conformation, this number is further reduced to $2^{5}$ conformers. Most of the conformations are either incompatible with the periodic boundary conditions, or have to significantly twist out of the plane to accommodate them, hence only a few configurations were suitable.

To select the suitable conformers, all distinct (32) molecular conformations were optimized in one-dimensional periodic boundary conditions using the B3LYP functional and the 6-311g(d) basis set. Four practically planar conformations with alkyl side chains on one side of the backbone are shown in Fig. 5C. Out of these four (labelled a,b,c,d in the Fig. 5C), one can construct the proposed unit cell using the period-matching pairs, such as $a-a, a-b, b-b, c-c, c-d$, or $d-d$. For the $a-a, b-b$ and $c-c$ pairs, one of the chains has to be flipped to avoid the overlap of side chains. One of these possible configurations is shown in the Fig. 4c.

Temperature dependence of PCDTBT thin films. To investigate the temperature evolution behaviour in detail, we have examined and analysed the scattering profiles along the $q_{z}$ axis and these were extracted from the $2 \mathrm{D}$ scattering patterns such as those shown in Fig. 2 (acquired every $20^{\circ} \mathrm{C}$ ). Representative scattering profiles, displayed as the lowest seven curves in Fig. 3 after subtraction of
Table 1 | The X-ray scattering-derived layer spacings and coherence lengths.

$\begin{array}{lccccccc}\text { Temperature }\left({ }^{\circ} \mathrm{C}\right) & 25 & 80 & 100 & 140 & 160 & 200 & 25 \dagger \\ \text { Layer spacing }(\AA) & 17.5 & 16.8 & 17.2 & 20.8 & 20.2 & 19.6 & 18.9 \\ \text { Coherence length }(\AA) & 27 & 30 & 32 & 78 & 107 & 123 & 185\end{array}$

The layer spacings and coherence lengths obtained by fitting the scattering profile along $q_{z}$ axis at various temperatures. After thermal treatment, the temperature is labelled as $25 \dagger$.

the diffuse signal, were obtained slightly off-axis to minimize the specular diffuse background. The Gaussian model fits, dashed lines displayed in Fig. 3, provide an excellent representation of the scattering profiles. The resulting fitting parameters are summarized in Table 1 , where the layer spacing $\left(a_{0}\right)$ was calculated from the Gaussian's centre $\left(q_{0}\right)$ by $a_{0}=2 \pi / q_{0}$ and the coherence length $(\xi)$, a value associated with the average grain size, was calculated from the standard deviation of the Gaussian fits $(\sigma)$ by $\xi=\pi / \sigma$. Over the temperature range from 25 to $100^{\circ} \mathrm{C}$, the layering peak is very broad and the analysis gives nearly temperature-independent coherence lengths and layer spacings, $\sim 30 \pm 3$ and $\sim 17.1 \pm 0.4 \AA$, respectively. Here the coherence length is only a few layers thick, similar to that of a liquid, and supports the designation that the pre-annealed sample is amorphous ${ }^{14}$. Despite an increase in the scattering intensity with increasing temperature below $100^{\circ} \mathrm{C}$, there is no change in the overall structural motif. However, at $140^{\circ} \mathrm{C}$, above the glass-transition temperature, the layering peak position is shifted to $0.302 \AA^{-1}$. This large shift in the layer spacing, $\sim 3 \AA$, is too large to be attributed to a simple lattice expansion, but rather it corresponds to a phase change. Further, this peak has narrowed corresponding to a coherence length of $78 \AA$. The second-order peak appears at $160^{\circ} \mathrm{C}$ and the third-order peak appears at $200^{\circ} \mathrm{C}$. By $200^{\circ} \mathrm{C}$, the scattering profiles have sharpened further and the coherence length reaches $123 \AA$ corresponding to about 6 layers, still about a factor of two less correlated than for P3HT at the same temperature ${ }^{24}$. The first order peak shifts slightly to higher $q_{z}$ when the temperature is raised from 140 to $200^{\circ} \mathrm{C}$, corresponding to a lattice contraction. This contraction may result from the ability of the layers to pack more efficiently in the crystalline phase. On cooling to $25^{\circ} \mathrm{C}$, the scattering patterns, Fig. 2 d, remained the same as at $200^{\circ} \mathrm{C}$ despite the $3.6 \%$ lattice contraction, exemplified by the shift in the peak positions, Fig. 3 (orange and yellow). This is much less than the $7 \%$ contraction observed for P3HT over this same temperature range ${ }^{25}$. 


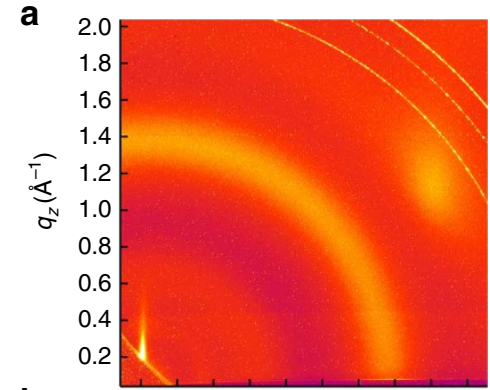

b

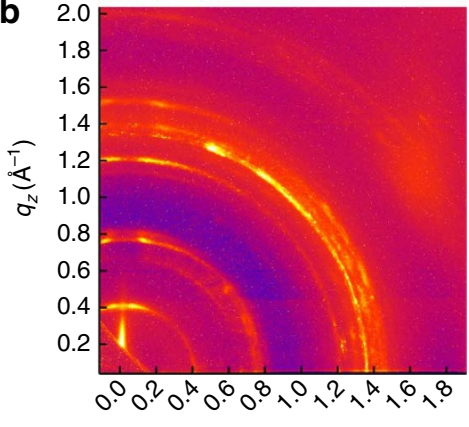

$q_{r}\left(\AA^{-1}\right)$

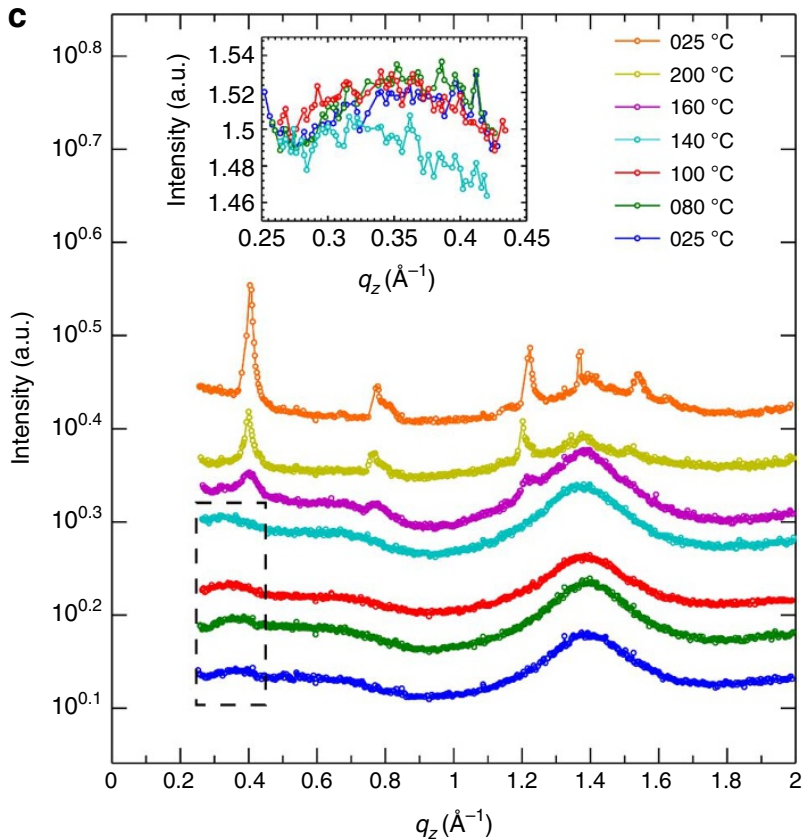

Figure 6 | GIWAXS images for PCDTBT blended with PCBM. The GIWAXS images are obtained at (a) $25^{\circ} \mathrm{C}$, before annealing, and (b) $25^{\circ} \mathrm{C}$, after annealing. (c) The corresponding scattering profiles along $q_{z}$. The inset shows the region of interest near $0.36 \AA^{-1}$, at temperatures at $1400^{\circ} \mathrm{C}$ and below. The blue and orange $25^{\circ} \mathrm{C}$ curves are measured before and after thermal treatment, respectively.

We attribute this larger P3HT coefficient to the higher volume fraction of alkyl chains in P3HT.

We have also examined the thermal behaviour of the off-axis peaks. On cooling to $25^{\circ} \mathrm{C}$, we observe no significant change in the $q_{r}$ positions $\left(0.150 \AA^{-1}\right)$ of the peaks. Thus, the scattering profiles of the off-axis peaks along $q_{z}$ are obtained from the $2 \mathrm{D}$ scattering images at $q_{r}=0.150 \AA^{-1}$, shown by the top two curves in Fig. 3 . The thermal behaviours of the off-axis peaks are very similar to the onaxis peaks. Together, our results show that the thermal expansion is along the layering direction and not along the backbone direction.

X-ray scattering studies of PCDTBT/PCBM blends. Figure 6 shows GIWAXS images of thin films of PCDTBT blended with PCBM at (a) $25^{\circ} \mathrm{C}$ before annealing, (b) $25^{\circ} \mathrm{C}$ after annealing and (c) the scattering profiles along $q_{z}$ at different temperatures. The 1:4 ratio of PCDTBT to PCBM is identical to that used in highefficiency bulk heterojunction devices, reported previously ${ }^{12,13}$. Because of the high concentration of PCBM, the amorphous PCBM ring at around $1.4 \AA^{-1}$ dominates the scattering image, whereas the weak layering and $\pi-\pi$ stacking peaks of the polymer are barely visible at $25^{\circ} \mathrm{C}$, before annealing (Fig. 6a).

After annealing, shown in Fig. 6b, the sharp peaks observed are from the crystallization of PCBM, consistent with previous results ${ }^{26}$. The scattering profiles along $q_{z}$ (Fig. $6 \mathrm{c}$ ) reveal details of the temperature-dependent structural evolution. Below $140^{\circ} \mathrm{C}$, the lowest order-layering peak is very weak (see inset) but it is located at $\sim 0.36 \AA^{-1}$, the same position as in the pure PCDTBT bilayer phase. This suggests the appearance of the bilayer phase in the blend material below $140^{\circ} \mathrm{C}$. The other crystalline peaks that indicate long-range order are not observed in the blends. At temperatures above $160^{\circ} \mathrm{C}$, a pronounced layering peak emerges in the blend corresponding to a $\sim 15.7 \AA$ layer spacing, too small for the bilayer phase. At these elevated temperatures, the rings of scattering from the PCBM are no longer isotropic after annealing suggesting orientational order that maybe induced by the PCDTBT crystallinity. This might correspond to a mixed PCDTBT/PCBM phase, such as observed for PBTTT blends ${ }^{27}$. Thus, our results suggest that a disordered phase with a local bilayer motif may account for the excellent cell performance observed in the blends below $140{ }^{\circ} \mathrm{C}$.

\section{Discussion}

To our knowledge, our proposed backbone pairing mode, composed of side-to-side backbones has not been previously reported for conjugated polymers. The physical motivation for the formation of the bilayer polymer backbone model can be found in block copolymers, polymers with a regular series of two different blocks. If the two blocks are relatively immiscible with each other, then the system undergoes a nanophase separation between the two blocks. Depending on the strength of the repulsive interactions, this may lead to lamellar, spherical or hexagonally packed phases ${ }^{28}$. Phospholipids also form a bilayer motif to nanophase separate their ionic (hydrophilic) and alkyl (hydrophobic) regions. For conjugated polymers, the alkyl regions would also like to phase separate from the backbone and for polymers such as regioregular P3HT; this leads to monolayer lamellar ordering, in part, because the alkyl chains must alternate from either side due to steric constraints. As, for PCDTBT, the alkyl chains are all anchored on the same segment, this allows the alkyl-free side of the PCDTBT backbones to pair. This arrangement is favoured, because it minimizes the interfacial energy between the nonpolar alkyl side chains and the polar backbones.

Developing structure-function relationships, in conjugated polymers, is essential for improving device performance in organic photovoltaic devices ${ }^{29}$. Our results show that one of the best performing device materials, PCDTBT, exhibits a bilayer structural motif described by conjugated backbone pairs with the alkyl chains filling the interstitial regions between the pairs. The bilayer motif may result from the lone attachment site of the alkyl chains on the backbone and it seems to be an equilibrium property of PCDTBT, because it is found in films at high temperature and at room temperature after annealing. The broad diffraction peaks observed in films before annealing, both in the pure material and in the blends, indicates amorphous-like behaviour and suggests a random 
network of bilayer pairs. Under these conditions, excellent device performance is observed. A better understanding of the bilayer motif in conjugated polymers and its relationship to device performance, especially in other materials, may aid in the design of new high-performance polymers for the next generation of transistor, light-emitting diode, and solar-cell applications.

\section{Methods}

Preparation of PCDTBT thin films. The PCDTBT, supplied by Konarka, has $M_{w} \sim 100 \mathrm{k}$, a polydispersity index of 2.75 , and a glass-transition temperature of $130^{\circ} \mathrm{C}$, consistent with previous measurements ${ }^{16}$. The thin films were prepared by blade-coating $8 \mathrm{mg} \mathrm{ml}^{-1}$ PCDTBT in 1,2-dichlorobenzene (oDCB) at $80^{\circ} \mathrm{C}$ on silicon wafers.

X-ray scattering measurements. The GIWAXS experiments were performed at the X9 undulator beamline at the National Synchrotron Light Source, Brookhaven National Laboratory, where the $14.0 \mathrm{keV}$ photons $(\lambda=0.0886 \mathrm{~nm})$ are focused to a spot with a height of $80 \mu \mathrm{m}$ and a width of $200 \mu \mathrm{m}$, at the sample position. The $\mathrm{X}$-ray incident angle was set to $\sim 0.15^{\circ}$, an angle above the critical angle of the polymer so as to assure a full penetration into the film. The diffraction images, such as those shown in Fig. 2, reveal the sample's out-of-plane structure (normal to the substrate) along vertical axis $\left(q_{z}\right)$ and in-plane structure (parallel to the substrate) along horizontal axis $\left(q_{r}\right)$. The scattering/sample chamber vacuum was maintained at $\sim 10^{-2}$ torr at all temperatures to reduce thermal sample degradation, beam damage, diffuse scattering and X-ray absorption. We have accounted for the intersection of Ewald sphere and reciprocal space in the extracted scattering profiles and the measured peak positions.

FTIR spectra. The aliphatic region of the FTIR spectra for PCDTBT, both as-cast and annealed, is shown in Supplementary Figure S1. PCDTBT was cast on undoped, zone-cast, double-side polished silicon wafers with resistivity $>6,000 \mathrm{ohms} \mathrm{cm}^{-1},<100>$ orientation, $380-410 \mu \mathrm{m}$ thickness, (WRS Materials). Spectra were collected in transmission mode at Brewster's angle $\left(\Theta=16^{\circ}\right)$ with a DTGS detector, using a Nicolet model 6,700 FTIR spectrometer (ThermoFisher) equipped with a KRS-5 polarizer (Spectra-Tech). Each spectrum represents the sum of 256 individual spectra, which were subjected to background correction, relative to a representative blank silicon chip. The corrected spectra were then fit to a second-order baseline and the peak locations and widths were modelled with a combination of Lorentzians using spectral analysis macros written by B. and P.U.P.A. Gilbert for the software package IGOR Pro 6 (Wavemetrics) $^{30}$. To determine the exact peak positions of each vibrational mode, we modelled the observed spectra with a combination of six Lorentzians ${ }^{21}$. Most relevant to this work was the peak position of the methylene anti-symmetric stretch at $2,928 \mathrm{~cm}^{-1}$. For extended alkyl chains, this vibrational mode is known to be sensitive to the degree of gauche defects present in the alkyl chain ${ }^{22}$. The position of this peak in the as-cast spectra is consistent with a 'liquid-like' alkyl side-chain (that is, one with a significant number of gauche defects). On thermal annealing, this peak position does not change, suggesting that the side-chains of PCDTBT exist in a disordered state, independent of the crystallinity of the backbones.

\section{References}

1. Sirringhaus, H. et al. Two-dimensional charge transport in self-organized, high-mobility conjugated polymers. Nature 401, 685-688 (1999).

2. McCulloch, I. et al. Liquid-crystalline semiconducting polymers with high charge-carrier mobility. Nature Mater. 5, 328-333 (2006).

3. Yan, H. et al. A high-mobility electron-transporting polymer for printed transistors. Nature 457, 679-686 (2009).

4. Winokur, M. J. \& Chunwachirasiri, W. Nanoscale structure-property relationships in conjugated polymers: Implications for present and future device applications. J. Polym. Sci B Polym. Phys. 41, 2630-2648 (2003).

5. Heeger, A. J. Semiconducting polymers: the third generation. Chem. Soc. Rev. 39, 2354-2371 (2010).

6. Coffin, R. C., Peet, J., Rogers, J. \& Bazan, G. C. Streamlined microwave-assisted preparation of narrow-bandgap conjugated polymers for high-performance bulk heterojunction solar cells. Nat. Chem. 1, 657-661 (2009).

7. Szarko, J. M. et al. When function follows form: Effects of donor copolymer side chains on film morphology and BHJ solar cell performance. Adv. Mater 22, 5468-5472 (2010).

8. Yang, L., Zhou, H. \& You, W. Quantitatively analyzing the influence of side chains on photovoltaic properties of polymer-fullerene solar cells. J. Phys. Chem. C 114, 16793-16800 (2010).

9. Shi, Q. et al. Side chain engineering of copolymers based on bithiazole and benzodithiophene for enhanced photovoltaic performance. Macromolecules $\mathbf{4 4}$, $4230-4240$ (2011).

10. Gadisa, A. et al. Effect of alkyl side-chain length on photovoltaic properties of poly(3-alkylthiophene)/PCBM bulk heterojunctions. Adv. Funct. Mater. 19, 3300-3306 (2009).
11. Blouin, N., Michaud, A. \& Leclerc, M. A low-bandgap poly(2,7-carbazole) derivative for use in high-performance solar cells. Adv. Mater. 19, 2295-2300 (2007).

12. Park, S. H. et al. Bulk heterojunction solar cells with internal quantum efficiency approaching 100\%. Nat. Photon 3, 297-302 (2009).

13. Sun, Y. et al. Efficient, air-stable bulk heterojunction polymer solar cells using moox as the anode interfacial layer. Adv. Mater. 23, 2226-2230 (2011).

14. Cho, S. et al. A thermally stable semiconducting polymer. Adv. Mater. 22, 1253-1257 (2010).

15. Peters, C. H. et al. High efficiency polymer solar cells with long operating lifetimes. Adv. Energy Mater. 1, 491-494 (2011).

16. Blouin, N. et al. Toward a rational design of poly (2,7-carbazole) derivatives for solar cells. J. Am. Chem. Soc. 130, 732-742 (2008).

17. Prosa, T. J., Winokur, M. J., Moulton, J., Smith, P. \& Heeger, A. J. X-ray structural studies of poly(3-alkylthiophenes): an example of an inverse comb. Macromolecules 25, 4364-4372 (1992).

18. Chang, J.- F. et al. Enhanced mobility of poly(3-hexylthiophene) transistors by spin-coating from high-boiling-point solvents. Chem. Mater. 16, 4772-4776 (2004).

19. Park, Y. D. et al. Solubility-controlled structural ordering of narrow bandgap conjugated polymers. Adv. Energy Mater. 1, 63-67 (2011).

20. Rogers, J. T., Schmidt, K., Toney, M. F., Kramer, E. J. \& Bazan, G. C. Structural order in bulk heterojunction films prepared with solvent additives. Adv. Mater. 23, 2284-2288 (2011).

21. Gurau, M. C. et al. Measuring molecular order in poly(3-alkylthiophene) thin films with polarizing spectroscopies. Langmuir 23, 834-842 (2007).

22. Snyder, R. G., Strauss, H. L. \& Elliger, C. A. Carbon-hydrogen stretching modes and the structure of $\mathrm{n}$-alkyl chains 1 . long, disordered chains. J. Phys. Chem. 86, 5145-5150 (1982).

23. Frisch, M. J. et al. Gaussian 09 Revision A. 1 (Gaussian Inc., Wallingford CT, 2009).

24. Hlaing, H. et al. Nanoimprint-induced molecular orientation in semiconducting polymer nanostructures. ACS Nano 5, 7532-7538 (2011).

25. Lilliu, S. et al. Dynamics of crystallization and disorder during annealing of P3HT/PCBM bulk heterojunctions. Macromolecules 44, 2725-2734 (2011).

26. Yang, X. et al. Crystalline organization of a methanofullerene as used for plastic solar-cell applications. Adv. Mater. 16, 802-806 (2004).

27. Mayer, A. C. et al. Bimolecular crystals of fullerenes in conjugated polymers and the implications of molecular mixing for solar cells. Adv. Funct. Mater. 19, 1173-1179 (2009).

28. Bates, F. \& Fredrickson, G. Block copolymer thermodynamics: Theory and experiment. Annu. Rev. Phys. Chem. 41, 525-557 (1990).

29. Brabec, C. J., Heeney, M., McCulloch, I. \& Nelson, J. Influence of blend microstructure on bulk heterojunction organic photovoltaic performance. Chem. Soc. Rev. 40, 1185-1199 (2011).

30. P.U.P.A. Gilbert 'GG Macros', http://home.physics.wisc.edu/gilbert/ (2012).

\section{Acknowledgements}

This research is supported by the US. Department of Energy, Basic Energy Sciences, by the Materials Sciences and Engineering Division (X.L, H.H. D.G. \& B.O.) which is supported under Contract No. DE-AC02-98CH10886 and by Konarka Technologies. This work was partially supported by Energy Laboratory Research and Development Initiative at Brookhaven National Laboratories. This work was also supported by the DFG program IRTG 1404, DFG grant SPP 1355, and BMBF grant MESOMERIE. The authors wish to thank Mario Leclerc, Gilles Dennler, Barney Grubbs, Kevin Yager and Chuck Black for valuable discussions.

\section{Author contributions}

X.L., H.H. and B.M.O. carried out the x-ray measurements. X.L., B.M.O. and W.H.J developed the bilayer model. J.P. was responsible for preparing the samples. D.S.G. carried out the FTIR measurements. D.A. and K.K. carried out the ab initio calculations All authors contributed to the editing of the manuscript.

\section{Additional information}

Supplementary Information accompanies this paper at http://www.nature.com/ naturecommunications

Competing financial interests: The authors declare no competing financial interests.

Reprints and permission information is available online at http://npg.nature.com/ reprintsandpermissions/

How to cite this article: Lu, X. et al. Bilayer order in a polycarbazole-conjugated polymer. Nat. Commun. 3:795 doi: 10.1038/ncomms1790 (2012). 